PFC/JA-90-5

COMPUTER SIMULATION OF RELATIVISTIC

MULTIRESONATOR CYLINDRICAL MAGNETRONS

by
Hej-Wai Chan
Chiping Chen
onald C. Davidson

February, 1990

This research was supported in part by SDIO/IST under the managment of Harry Diamond Laboratories, in part by the Department of Energy High Energy Physics Division, and in part by the Naval Surface Warfare Center. 


\title{
COMPUTER SIMULATION OF RELATIVISTIC
}

\section{MULTIRESONATOR CYLINDRICAL MAGNETRONS}

\author{
Hei-Wai Chan, Chiping Chen and Ronald C. Davidson \\ Plasma Fusion Center \\ Massachusetts Institute of Technology \\ Cambridge, MA 02139
}

\begin{abstract}
$\underline{\text { ABSTRACT }}$
The relativistic multiresonator magnetron is analyzed in cylindrical geometry, using the two-dimensional particle-in-cell simulation code MAGIC. Detailed comparisons are made between the simulation results and the experiments by Palevsky and Bekefi [Phys. Fluids 22, 986 (1979)] using the A6 magnetron configuration. Within a constant scale factor, the computer simulations show a similar dependence of microwave power on magnetic field, with dominant excitations in the $\pi$ and $2 \pi$ modes. In the preoscillation regime, the electron flow in the simulations differs substantially from the ideal Brillouin flow model. In the nonlinear regime, the saturation is dominated by the formation of spokes.

PACS numbers: $52.25 . \mathrm{Wz}, 52.75 . \mathrm{PV}$
\end{abstract}


In relativistic magnetrons, ${ }^{1-6}$ pulsed high-voltage diodes (operating in the several hundred $\mathrm{kV}$ to $\mathrm{MV}$ range, say) are used to generate microwaves at gigawatt power levels. Although magnetrons are widely used as microwave sources, a fundamental understanding of the underlying interaction physics is still being developed, 7 particularly in the nonlinear regime. Much of the theoretical challenge in describing multiresonator magnetron operation arises from the complexity introduced by the corrugated anode boundary ${ }^{8}$ and the fact that the electrons emitted from the cathode interact with the electromagnetic waves in the anode-cathode gap in a highly nonlinear way. This is manifest through strong azimuthal bunching of the electrons and the formation of large-amplitude "spokes" in the electron density. In this regard, computer simulation studies ${ }^{9-11}$ provide a particularly valuable approach to analyze the interaction physics and nonlinear electrodynamics in magnetrons.

In this letter, we summarize recent computer simulations of the multiresonator A6 magnetron configuration ${ }^{3}$ using the twodimensional $(\partial / \partial z=0)$ particle-in-cell code MAGIC. ${ }^{12}$ The code includes cylindrical effects, and relativistic and electromagnetic effects in a fully self-consistent manner. Unlike previous computer simulations, ${ }^{9-11}$ the magnetron oscillations are excited from noise, i.e., without preinjection of a finite-amplitude rf signal or preferential excitation of $2 \pi$-mode or $\pi$-mode oscillations. In addition, the present simulations are carried out in cylindrical. rather than planar 9,10 magnetic geometry. Figure 1 shows the cross section of the A6 magnetron. ${ }^{3}$ The field-emission cathode is located at radius $a=1.58 \mathrm{~cm}$. The inside radius of the anode block is $b=2.11 \mathrm{~cm}$, and six vane-type resonators with outer 
radius $\mathrm{d}=4.11 \mathrm{~cm}$ are used, with angle $\psi=20^{\circ}$ subtended by the resonators on axis. The applied magnetic field $B_{f} \hat{e}_{z}$ ranges from 4-10 kG in typical operation. ${ }^{3}$ The axial length of the anode block is $L=7.2 \mathrm{~cm}$, and the operating voltage is $300-400 \mathrm{kV}$. For transverse electric (TE) excitations with $\delta_{\sim}^{\mathrm{E}}$ perpendicular to $B_{f} \hat{\mathrm{e}}_{z}$ and $\delta \underset{\sim}{B}$ parallel to $B_{f} \hat{e}_{z}$, the vacuum electric field pattern in Fig. 1 is such that $\delta E_{\theta}$ is in phase in adjacent resonators for the $2 \pi$ mode, whereas $\delta E_{\theta}$ is out of phase in adjacent resonators for the $\pi$ mode .

In the simulations, Maxwell's equations and the particle orbit equations are solved relativistically and electromagnetically, using (typically) more than 3000 macroparticles and a nonuniform, two-dimensional grid consisting of approximately 3000 cells. When a voltage $V_{D}(t)$ is applied across the diode in Fig. 1 , the electrons are emitted from the cathode through a space-charge-limited emission process in which the instantaneous electric field normál to the cathode surface vanishes. The radial momenta of the emitted electrons are randomly distributed from 0 to $0.02 \mathrm{mc}$. On the other hand, electrons are absorbed by the anode or cathode whenever they strike the surface. The simulations are carried out with one open resonator, which is modeled by a dispersive window placed along the dashed line in Fig. 1 at $r=d=4.11 \mathrm{~cm}$. At the window, the boundary condition is such that most of the electromagnetic wave energy is absorbed, while a small fraction of the wave energy is reflected back into the cavity.

A quasi-static model is used in the simulations to describe the high-voltage pulse applied to the diode. In such a model, the diode voltage is given by $v_{D}(t)=z(t) v_{0}(t) /\left[z_{0}+z(t)\right]$, where $v_{0}(t)$ is the voltage pulse provided by the power supply, $z_{0}=$ 
const. is the power supply impedance, and $z(t)$ is the magnetron impedance. In the simulations, $v_{0}(t)$ is assumed to have the form

$$
v_{0}(t)=\left\{\begin{array}{l}
0, t<0, \\
\left(t / t_{0}\right) v_{m}, 0 \leq t<t_{0}, \\
v_{m}, t \geq t_{0},
\end{array}\right.
$$

where $t_{0}$ and $v_{m}$ are the rise time and maximum value of the voltage pulse, respectively. The rise time assumed in the simulations is $t_{0}=4.0 \mathrm{~ns}$, corresponding to the experimental value. ${ }^{3}$ Although the applied voltage pulse in the simulations is described by Eq.(1), all extraordinary-mode rf excitations ( $\delta \underset{\sim}{B}=\delta B_{z} \underset{\sim}{\hat{e}_{z}}$ and $\delta \underset{\sim}{E}=$ $\left.\delta E_{r} \hat{e}_{r}+\delta E_{\theta} \hat{\hat{e}}_{\theta}\right)$ are treated fully electromagnetically. Typical numerical results are presented in Figs. 2-4 for the ideal case where $z_{0}=0$ and $v_{D}(t)=v_{0}(t)$.

Figure 2 shows the time history of the integrated rf field profile $V_{\theta}(t)=\int_{P_{1}}^{P_{2}} d \theta r_{\ell} \delta E_{\theta}\left(r_{l}, \theta, t\right)$ and the magnitude of the Fourier transform of $v_{\theta}(t)$, denoted by $\left|\hat{v}_{\theta}(f)\right|$, for the choice of system parameters $B_{f}=7.2 \mathrm{kG}, V_{m}=350 \mathrm{kV}$ and $t_{0}=4.0 \mathrm{~ns}$. Here, the integration path corresponds to the dotted line in Fig. 1 from $P_{1}$ to $P_{2}$ at $r=r_{l}=3.7 \mathrm{~cm}$. In Fig. $2(a)$, the nonlinear saturation of the magnetron oscillations occurs at $t \simeq 10 \mathrm{~ns}$, where the ratio of the saturated amplitude $V_{\theta s}$ and the applied diode voltage $V_{D}=$ $v_{m}$ is $v_{\theta s} / V_{D}=0.85$. In Fig. $2(b)$, the two distinct peaks at the frequencies $f=2.0 \mathrm{GHz}$ and $f=4.0 \mathrm{GHz}$ correspond to $\pi$-mode and $2 \pi$-mode oscillations, respectively. The $2 \pi$-mode oscillation frequency $f=4.0 \mathrm{GHz}$ is $14 \%$ lower than the frequency $f=4.55 \mathrm{GHz}$ observed in the experiment, 3 which may be due to the absence of finite-axial-length effects in the simulations (where $\partial / \partial z=0$ 
is assumed). Although both excitations have nearly the same amplitude in Fig. $2(b)$, the (higher frequency) $2 \pi$ mode delivers more rf power than the $\pi$ mode.

By evaluating the area-integral of the outward poynting flux $(c / 4 \pi) \delta E_{\theta} \delta B_{z}$ over the surface of the dispersive window at $r=d$ $=4.11 \mathrm{~cm}$ in Fig. 1 , the peak $\mathrm{rf}$ power output in the simulations is calculated for various values of the applied magnetic field $B_{f}$. The dependence of the normalized rf power on magnetic field is shown in Fig. 3. Here, the dots correspond to the experimental values, ${ }^{3}$ and the triangles are obtained from the simulations with $z_{0}=0$. In Fig. 3, the normalization is chosen such that the maximum values of the $r f$ power in both the simulations and the experiment are equal to unity. The actual value of the maximum rf output per unit axial length in the simulations is $4.3 \mathrm{GW} / \mathrm{m}$. For the A6 magnetron, with axial length $\mathrm{L}=7.2 \mathrm{~cm}$, this corresponds to $P=0.3 \mathrm{G}$, which is somewhat less than the maximum $r f$ power $P=0.45 \mathrm{GW}$ measured in the experiment. ${ }^{3}$ (The values of power quoted here are rms values.) Apart from a constant scale factor, it is evident from Fig. 3 that the simulations are in excellent agreement with the experimental results. The difference in scale factor may be due to the fact that a larger fraction of the $r f$ power in the simulations is reflected back into the cavity, and the effective $Q$-value in the simulations $(Q \sim 100)$ is greater than in the experiment $(Q \sim 20-40)$. As $B_{f}$ is decreased below $6 \mathrm{kG}$, crossing the Hull cut-off curve ${ }^{2}$ at a diode voltage corresponding to $v_{D}=350 \mathrm{kV}$, it is found that the $5 \pi / 3$-mode $(l=5)$ becomes the dominant of excitation in the simulations. 
In following the time evolution of the electron density $n_{e}(r, \theta, t)$ in the simulations, it is $\underset{2 \pi}{2}$ that the profile for the average density, $\left\langle n_{e}\right\rangle(r, t)=(2 \pi)^{-1} \int_{0} d \theta n_{e}(r, \theta, t)$, does not resemble that corresponding to Brillouin flow, ${ }^{2}$ even at early times ( $t<4 n s, s a y)$. In particular, the profile for $\left\langle n_{e}\right\rangle(r, t)$ decreases as $r$ increases from $r=a=2.11 \mathrm{~cm}$, and exhibits a long tail extending beyond the layer radius $r_{b}$ calculated from an ideal Brillouin flow model. ${ }^{2}$ Although the azimuthal bunching of the electrons is relatively small for times up to $4 \mathrm{~ns}$, by $t z$ 6 ns the system begins to enter a nonlinear regime characterized by spoke formation. Highly developed spokes are evident in Fig. 4(b) which shows density contour plots at $t=8 \mathrm{~ns}$ for the choice of system parameters $B_{f}=7.2 \mathrm{kG}, v_{m}=350 \mathrm{kV}$ and $t_{0}=$ $4 \mathrm{~ns}$. The spokes rotate in the azimuthal direction as the system evolves, and there is current flow from the cathode to the anode. At saturation, which occurs at $t \simeq 10 \mathrm{~ns}$, the time-averaged diode current per unit axial length is $I_{D} \simeq 100 \mathrm{kA} / \mathrm{m}$, and the amplitude of the integrated $r f$ field profile $\int_{a}^{b} d r \delta E_{r}(r, \theta, t)$ is comparable with the diode voltage $v_{D} \simeq v_{m}=350 \mathrm{kV}$.

To summarize, with regard to the dependence of if power on magnetic field, the simulation results are in excellent agreement with the experiment (within a constant scale factor). Also, in terms of $r f$ power output, the simulations confirm that the A6 magnetron oscillates preferentially in the $2 \pi$ mode. In the preoscillation regime, it is found that the electron flow differs substantially from Brillouin flow conditions. In the nonlinear regime, the saturation is dominated by the formation of spokes. The simulations also show that the magnetron performance and $x f$ 
power generation are degraded when the impedance $z_{0}$ of the external power supply is increased (in agreement with experiment) from the ideal value $z_{0}=0$. As a general conclusion, based on the results of this paper, it is expected that the MAGIC simulation code can be used as an effective tool for experimental magnetron design.

\section{ACKNOWLEDGMENTS}

The authors wish to thank George Bekefi, shien Chi Chen and George Johnston for helpful discussions, and Bruce Goplen and Jim MCDonald of Mission Research Corporation for consultations on the MAGIC simulation code. This research was supported in part by SDIO/IST under the management of Harry Diamond Laboratories, in part by the Department of Energy High Energy Physics Division, and in part by the Naval surface Warfare center. 


\section{REFERENCES}

1. J. Benford, in High-Power Microwave Sources, eds., V. Granatstein and I. Alexeff (Artech House, Boston, Massachusetts, 1987) p. 351 .

2. R.C. Davidson, Physics of Nonneutral Plasmas (Addison-Wesley, Reading, Massachusetts, 1990) Chapter 8.

3. A. Palevsky and G. Bekefi, Phys. Fluids $\underline{22}, 986$ (1979).

4. G. Bekefi and T.J. Orzechowski, Phys. Rev. Lett. 37, 379 (1976).

5. J. Benford, H.M. Sze, W. Woo, R.R. Smith, and B. Harteneck, Phys. Rev. Lett. 62,969 (1989).

6. A.G. Nokonov, I.M. Roife, Yu.M. Savel'ev, and V.I. Engel'ko, Sov. Tech. Phys. 32, 50 (1987).

7. Y.Y. Lau, in High-Power Microwave Sources, eds., V. Granatstein and I. Alexeff (Artech House, Boston, Massachusetts, 1987) p. 309.

8. H.S. Uhm, H.C. Chen, R.A. Stark, and H.E. Brandt, Proc. SPIE 1061, $170(1989)$.

9. S.P. Yu, G.P. Kooyers and O. Buneman, J. Appl. Phys. 36 , $2550(1965)$.

10. A. Palevsky, G. Bekefi, and A.T. Drobot, J. Appl. Phys. $\underline{52}$, $4938(1981)$.

11. A. Palevsky, et al., in High-Power Beams, eds., H.J. Doucet and J.M. Buzzi (Ecole Polytechnique, Palaiseau, France, 1981) p. 861 .

12. B. Goplen and J. MCDonald, private communication (1989). The MAGIC simulation code was developed by researchers at Mission Research Corporation. The simulation results presented in this paper use the code version dated 1988. 


\section{FIGURE CAPTIONS}

Fig. 1. Cross section of the $A 6$ magnetron used in the computer simulations. The rf power is partially absorbed by the dispersive window (dashed line) located at $r=d=$ $4.11 \mathrm{~cm}$ in the open resonator. An applied magnetic field $B_{f} \hat{e}_{z}$ points out of the page.

Fig. 2. Shown in Fig. $2(a)$ is the time history of the integrated $r f$ field profile $v_{\theta}(t)=\int_{P_{1}}^{P_{2}} d \theta r_{\ell} \delta E_{\theta}\left(r_{l}, \theta, t\right)$ obtained in the simulations at $\operatorname{radius}^{\mathbf{P}_{\mathbf{r}}}=\mathbf{r}_{\ell}=3.7 \mathrm{~cm}$ in the open resonator for the choice of system parameters $B_{f}=7.2 \mathrm{kG}, v_{m}=350 \mathrm{kV}, t_{0}=4.0 \mathrm{~ns}$, and $z_{0}=0$. Figure $2(b)$ shows the Fourier spectrum $\left|\hat{v}_{\theta}(f)\right|$ of the signal in Fig. 2(a). The two distinct peaks at $f=$ $2.0 \mathrm{GHz}$ and $f=4.0 \mathrm{GHz}$ correspond to $\pi$-mode $(l=3)$ and $2 \pi$-mode $(l=6)$ oscillations, respectively.

Fig. 3. Plots of the normalized peak rf power versus the applied magnetic field $B_{f}$. The dots correspond to the experimental results [A. Palevsky and G. Bekefi, Phys. Fluids 22 , $986(1979) \mathrm{J}$, and the triangles correspond to the simulation results for $v_{m}=350 \mathrm{kV}, t_{0}=4.0 \mathrm{~ns}$, and $\mathrm{z}_{0}=0$.

Fig. 4. Density contour plots for $n_{e}(r, \theta, t)$ obtained in the simulations at (a) $t=3.0 \mathrm{~ns}$ and (b) $t=8.0 \mathrm{~ns}$ for the same system parameters as in Fig. 2 . 


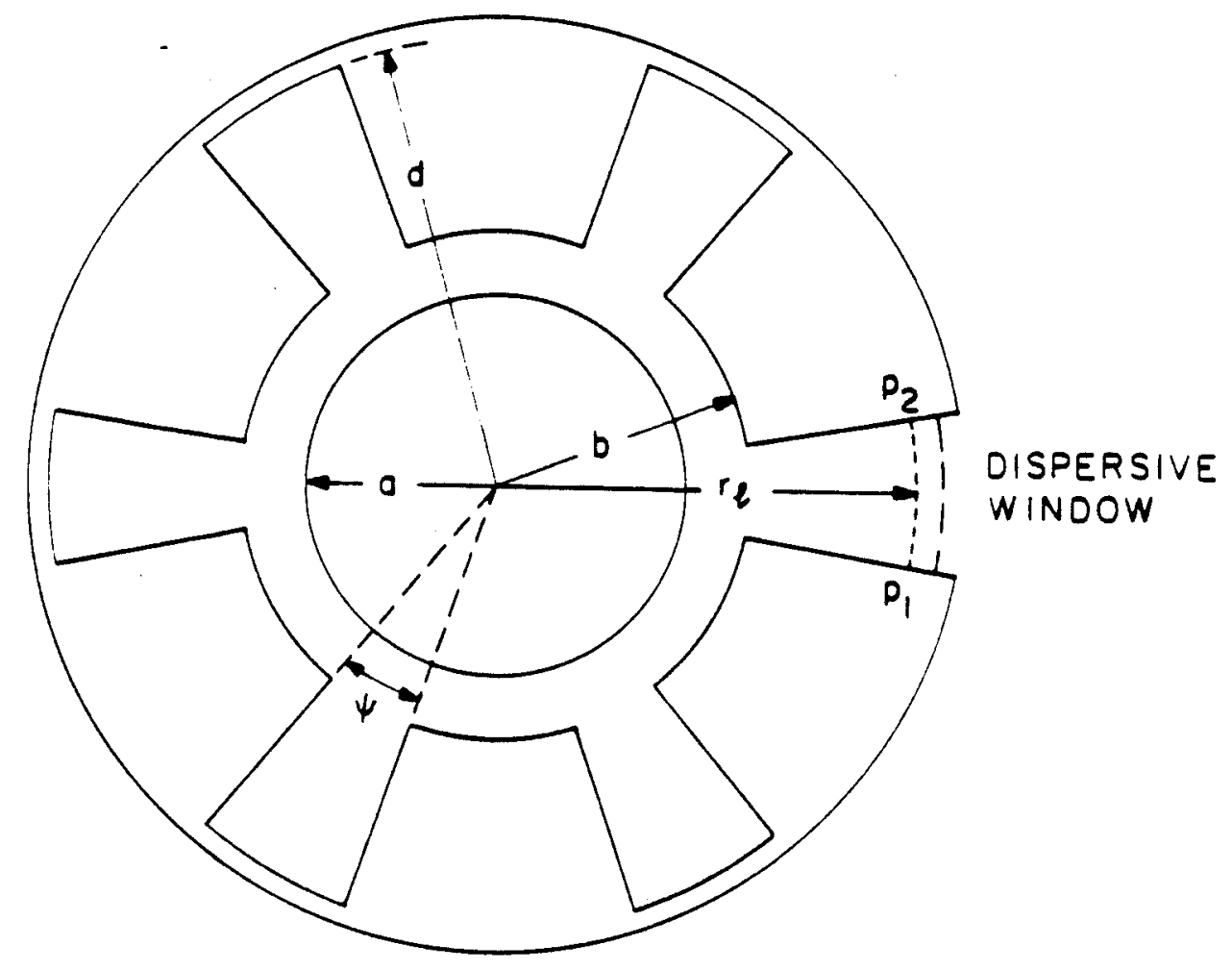

Fig. 1 

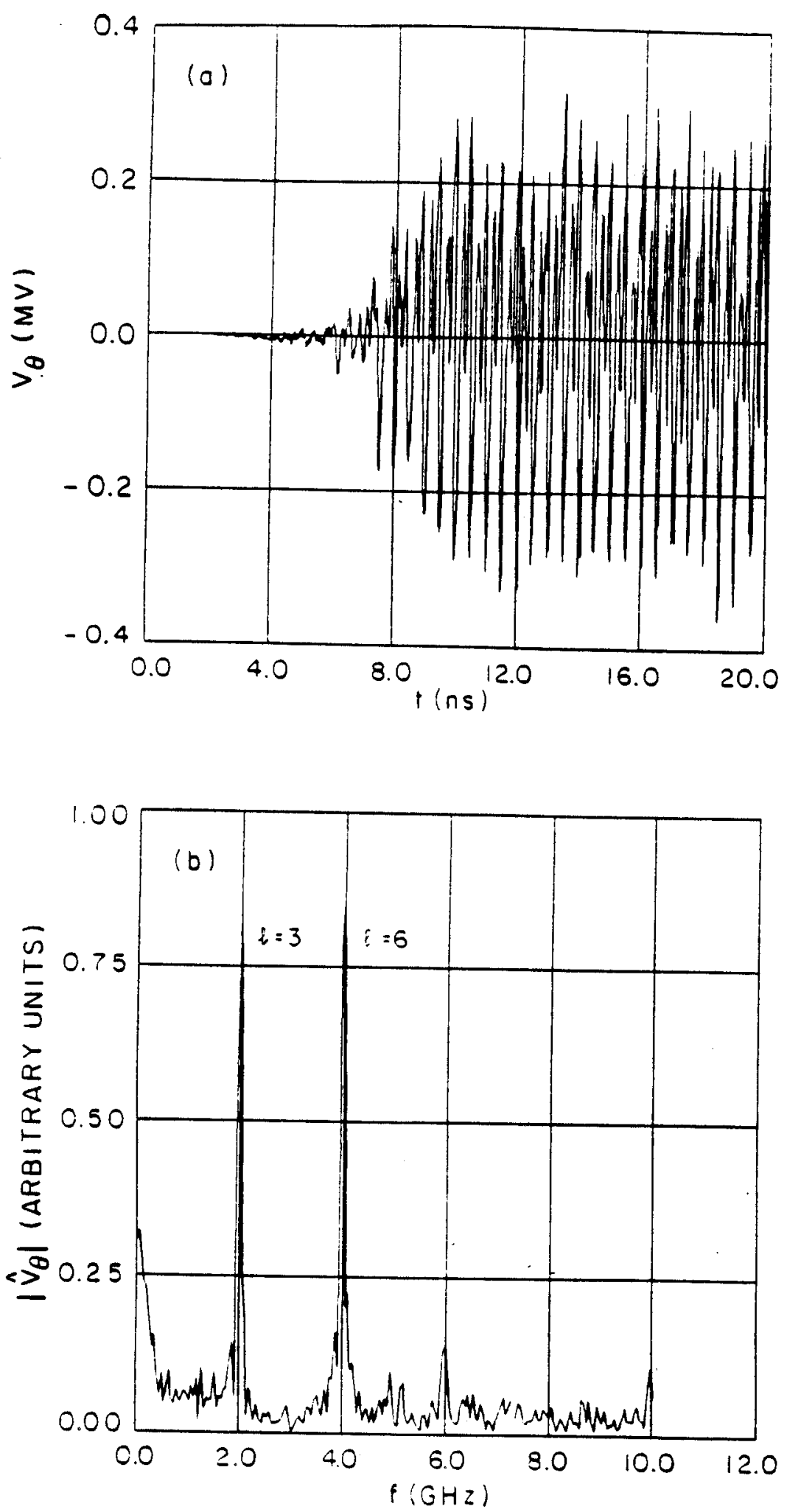

Fig. 2 


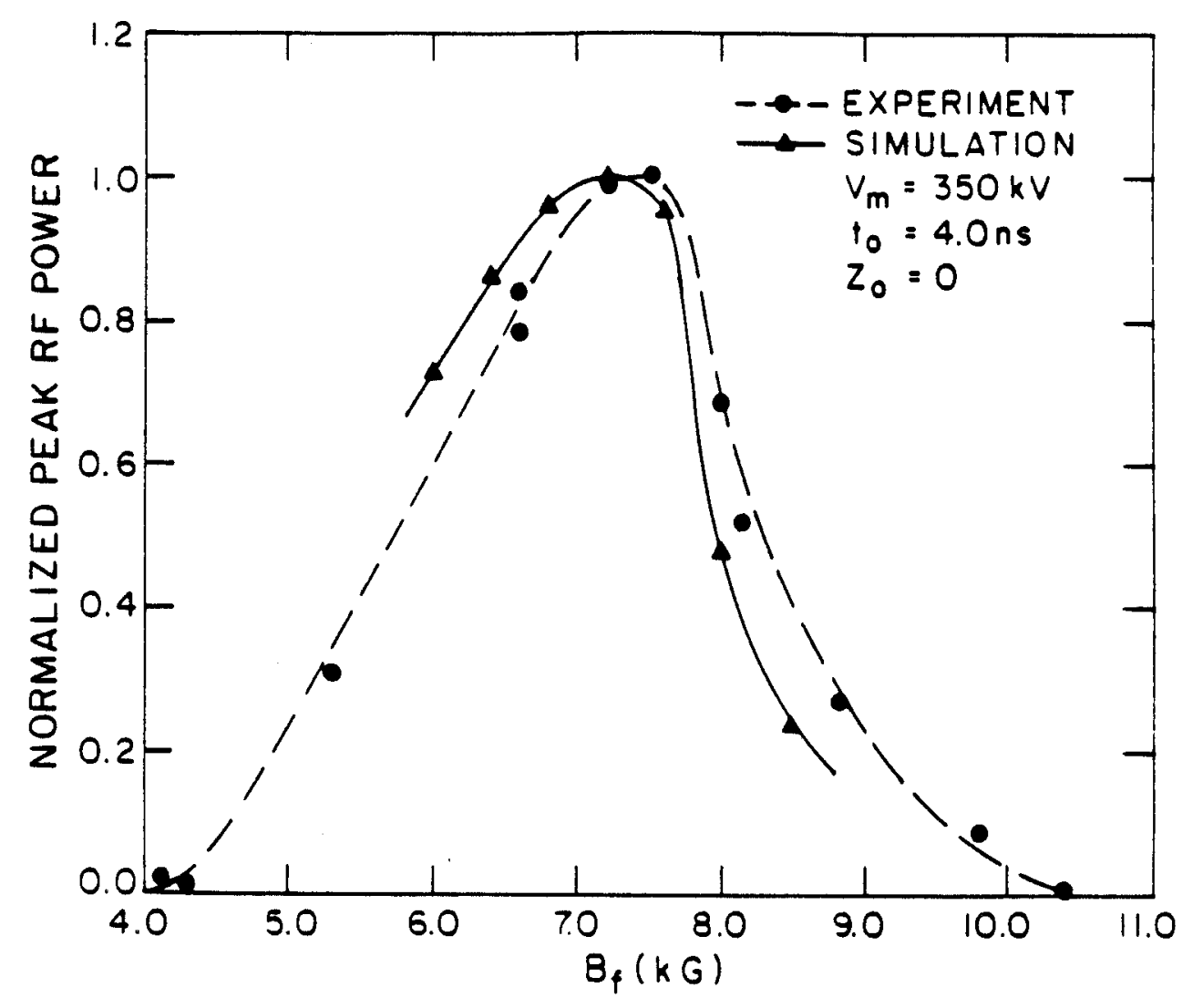

Fig. 3 

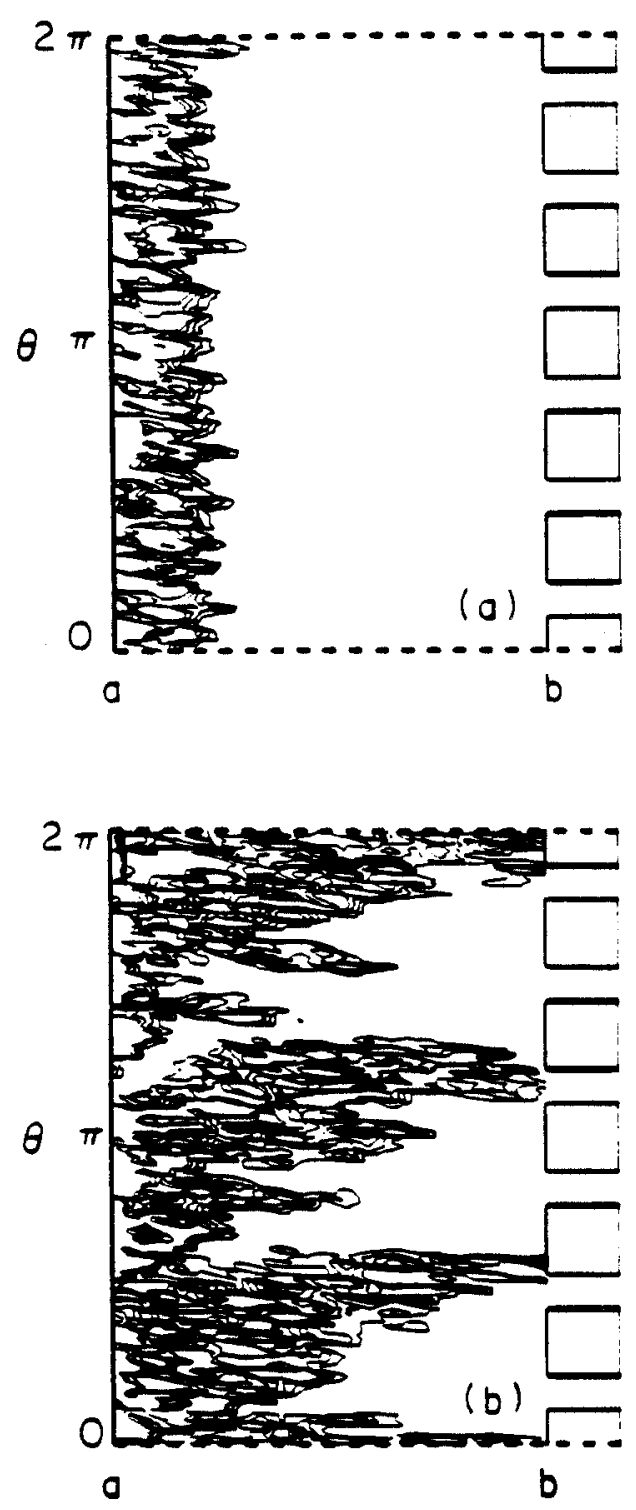

Fig. 4 